\title{
HOMOTOPY INVARIANTS IN DIFFERENTIAL GEOMETRY. I
}

\author{
BY \\ TADASHI NAGANO
}

Introduction. In this series of papers we will discuss homotopy invariants of differentiable maps $f: M \rightarrow N$ in various situations within the framework of differential geometry. We shall be particularly interested in the case where $f$ is an immersion. Our principle is simple. We use the fact that the pullback $f^{*} \omega$ of $\omega$ is a differentiable homotopy invariant where $\omega$ is an arbitrary cohomology class always over the real number field, $R$. We note if $F=M \times I \rightarrow N$ is a differentiable map with $f_{0}=f$, then $f^{*} \omega$ belongs to the same cohomology class as $f_{1}^{*} \omega$ where $I$ is the interval $[0,1]$ and $f_{t}$ is defined by $f_{t}(x)=F(x, t), x \in M, t \in I$.

There are many known examples beside various characteristic classes. To quote a few of them, let $f$ be an immersion of the two-torus $T^{2}$ into the complement of the diagonal set of the six dimensional euclidean space $R^{6}$ considered as $R^{3} \times R^{3}$; $f: T^{2} \rightarrow R^{3} \times R^{3}-\Delta$, where $\Delta$ is the diagonal set. The space $\boldsymbol{R}^{3} \times \boldsymbol{R}^{3}-\Delta$ is diffeomorphic with $R^{4} \times S^{2}$ where $S^{2}$ is the two-sphere. Thus, if $\omega$ denotes the volume element of $S^{2}$, $\omega$ becomes a 2-dimensional cohomology class of $R^{4} \times S^{2}$ by pulling back with the projection onto $S^{2}$ and gives us a homotopy invariant $f^{*} \omega$. Consider $T^{2}$ interpreted as the direct product $S^{1} \times S^{1}$ of circles and $f$ as the pair of two closed curves $c_{i}: S^{1} \rightarrow R^{3}$ without intersection. Then $f^{*} \omega$ is nothing but the linking number of two closed curves $c_{1}$ and $c_{2}$ (up to a universal constant multiple) according to Gauss [8]. Another (but somewhat more extraneous) example, due to J. H. C. Whitehead [12], is the Hopf invariant, $H(f)$, for a differentiable map $f: S^{2 n-1} \rightarrow S^{n}$. Again, denoting the volume element of the $n$-sphere $S^{n}$ by $\omega, H(f)$ is given by the closed form $\theta \wedge f^{*} \omega$ where $\theta$ is any $(n-1)$-form with $d \theta=f^{*} \omega$.

In this paper $f$ will be an isometric immersion of a compact oriented Riemannian manifold $M$ into a euclidean space $\boldsymbol{R}^{n+\nu}$. Since the cohomology groups of $N=\boldsymbol{R}^{n+v}$ are then trivial, our principle does not apply to $f$ directly, but we construct a manifold $B$ from $M$ and replace $f$ by a certain map $f^{B}: M \rightarrow B$. To do this, let us recall Hirsch's theorem [5] to the effect that the regular homotopy classes of the immersions $f: M \rightarrow N$ of any manifold $M$ into another manifold $N$ with $\operatorname{dim} N>\operatorname{dim} M$ are in a one-to-one correspondence with the homotopy classes of the crosssections $f^{B}: M \rightarrow B$ of a certain bundle $B$ over $M$, where a regular homotopy $F=M \times I \rightarrow N$ means one for which each $f_{t}$ is an immersion. Despite the triviality of the cohomology groups of $N=\boldsymbol{R}^{n+v}$ in the case above, we can expect to obtain homotopy invariants $f^{B^{*}} \theta$ corresponding to a cohomology class $\theta$ of $B$. In $\S 4$, we

Received by the editors February 12, 1969. 
will construct an important example of $f^{B^{*}} \theta$, which is expressed with the second fundamental form for $f$.

In differential geometry, manifolds usually carry an additional structure (like a Riemannian or Kählerian structure) other than the differentiable structure and one might wish to develop a restricted type of homotopy pertinent to that additional structure. To be more specific, in the case of the isometric imersions $f: M \rightarrow N$ of this paper, there will be some hope of having a differential form $\theta$ on $B$, not necessarily closed, such that $f^{B^{*}} \theta$ is an isometric homotopy invariant, meaning that $f_{t}^{B^{*}} \theta$ remains in one and the same cohomology class of $M$ as long as $f_{t}$ is an isometric immersion. $\theta$ should not be closed since otherwise $f^{B^{*}} \theta$ would be a mere regular homotopy invariant; and yet each $f_{t}^{B^{*}} \theta$ should be closed in order to give a cohomology class. This would be guaranteed if $\theta$ on $B$ is closed when restricted to each integral element $H$ of the differential system $\mathscr{H}$ on $B$ (see $\S 3$ ), the system intimately related to the given structure (the Riemannian metric in this paper) of $M$. For a description of $\mathscr{H}$, note that locally the integral manifolds of $\mathscr{H}$ are in a one-to-one correspondence with the isometric immersions $f$ (Proposition 3.2). In $\S 5$ we will construct examples of $\theta$ with the property we have just described. The result (Theorem 5.1) means that the integrals over $M$ of the symmetric functions of the principal curvatures of $f$ are isometric homotopy invariants for isometric immersions $f$ of $M$ as hypersurfaces. (See Remark 5.2 for a more intuitive geometric meaning of this result.)

$\$ \$ 1-3$ are preliminaries, the contents of which would be more or less known. In $\S 1$ we will construct $B$ for $M$ and $f^{B}$ for $f$ and give a condition that a section $s$ of $B$ must satisfy for $s$ to be some $f^{B}$ (Proposition 1.1). In $\$ 2$ we define differential forms necessary to describe any other forms and give the formulas for their exterior derivatives (i.e. the structure equations) using the integral geometric method developed by Chern and others. In $\S 3, \mathscr{H}$ will be defined and studied. $\$ \$ 4$ and 5 will be devoted to examples of the regular and the isometric homotopy invariants respectively. A number of remarks will be added without proofs mostly to explain geometric meanings, although they are logically redundant.

1. The bundle $B$ and the section $f^{B}$. $\quad M$ will always denote an oriented Riemannian manifold of dimension $n$. Let $B=B_{v}$ be the space of all linear isometries (=monomorphisms of metric vector spaces) $b: T_{x}(M) \rightarrow \boldsymbol{R}^{n+v}$ of the tangent spaces to $M$ at the points $x$ into the $(n+\nu)$-dimensional euclidean space $R^{n+v}$. In case $\nu=0$ we agree that the maps $b$ in $B$ preserve the orientation. We denote by $P$ the oriented orthonormal frame bundle, which is $\left\{b^{-1} \mid b \in B_{0}\right\}$ with a certain differentiable principal SO $(n)$-bundle structure. $\pi_{P}$ will denote the projection of $P$ onto $M . B$ becomes a differentiable fiber bundle in the following way. The projection $\pi_{B}: B \rightarrow M$ sends $b: T_{x}(M) \rightarrow R^{n+v}$ to $x \in M$. The (standard) fiber is the set of all linear isometries of $\boldsymbol{R}^{n}$ into $\boldsymbol{R}^{n+v}$, which, in case $\nu=0$, preserve the orientation, of the fiber $\pi_{B}^{-1}(0)$ over the origin 0 . This space, called a Stiefel manifold, will be 
denoted by $V_{n+v, n}$. Now the special orthogonal group $\mathrm{SO}(n)=V_{n, n}$ acts on $V_{n+v, n}$ to the right; each $g \in \mathrm{SO}(n)$ sends $v: \boldsymbol{R}^{n} \rightarrow \boldsymbol{R}^{n+v}$ to $v g=v \circ g: \boldsymbol{R}^{n} \rightarrow \boldsymbol{R}^{n}$ $\rightarrow R^{n+v}$. SO $(n)$ acts on $P$ also to the right; $g$ sends $p: R^{n} \rightarrow T_{x}(M)$ to $p g=p \circ g: R^{n} \rightarrow T_{x}(M)$. Thus, if one defines the map $\pi: P \times V_{n+v, n} \rightarrow B$ by $\pi(p, v)=v \circ p^{-1}: \boldsymbol{R}^{n} \rightarrow T_{x}(M) \rightarrow \boldsymbol{R}^{n+v}$, then $\pi$ becomes the principal map for the bundle so that (1) $\pi$ is surjective (or rather a submersion), (2) $\pi(p, v)$ equals $\pi\left(p^{\prime}, v^{\prime}\right)$ if and only if there exists some $g \in \mathrm{SO}(n)$ such that $p g=p^{\prime}$ and $v g=v^{\prime}$. The differentiable structure is introduced on $B$ so that $B$ becomes a differentiable fiber bundle. The group SO $(n+\nu)$ acts on $B_{v}$ to the left; each $a \in \mathrm{SO}(n+\nu)$ sends $b \in B$ to $a b=a \circ b: T_{x}(M) \rightarrow R^{n+v} \rightarrow R^{n+v}$. In this action SO $(n+v)$ leaves each fiber invariant. In particular SO $(n+\nu)$ acts on $V_{n+v, n}$ to the left and gives the identification $V_{n+v, n}=\mathrm{SO}(n+\nu) / \mathrm{SO}(\nu)$, where $\mathrm{SO}(\nu)$ is identified with $\left\{1_{n}\right\} \times \mathrm{SO}(\nu) \subseteq \mathrm{SO}(n+\nu)$ and $1_{n}$ is the $n \times n$ unit matrix.

Given an immersion $f: M \rightarrow R^{n+v}$, we will construct a cross-section $f^{B}: M \rightarrow B$ of the bundle $B$. The differential $f_{*}$, restricted to each tangent space $T_{x}(M)$, is a vector space monomorphism of $T_{x}(M)$ into $T_{f(x)}\left(\boldsymbol{R}^{n+v}\right)$ which we identify with the vector space $\boldsymbol{R}^{n+v}$ in a natural way. Next we factor this map $f_{*} \mid T_{x}(M)$ into the composite of the two maps:

$$
T_{x}(M) \stackrel{f_{*}}{\longrightarrow} f_{*}\left(T_{x}(M)\right) \stackrel{i}{\longrightarrow} R^{n+\imath}
$$

where $i$ is the inclusion map. And we modify $i$ to a linear isometry $\Gamma\left(f_{*}, x\right)$ by the Gram-Schmidt method of orthogonalization. Finally the composite $\Gamma\left(f_{*}, x\right) \circ f_{*}$ gives the desired $f^{B}(x) . f^{B}$ is clearly a differentiable section of $B$. We have $f^{B}(x)$ $=f_{*} \mid T_{x}(M), x \in M$, when and only when $f$ is an isometric immersion. Since the Gram-Schmidt method gives a retraction of the space of the linear morphisms onto that of the linear isometries and is homotopic with the identity in a canonical way, $f^{B}$ will vary continuously when $f$ does.

One might ask when a section $s: M \rightarrow B$ comes from an immersion $f$ to yield $s=f^{B}$. To answer this question, we introduce the canonical form $\left(\Omega^{\lambda}\right)_{1 \leqq \lambda \leqq n+\nu}$ of $B$. Each $\Omega^{\lambda}$ is a differential one-form on $B$, which assigns the $\lambda$ th component of the vector $b\left(\pi_{B^{*}}(X)\right)$ in $R^{n+v}$ to a tangent vector $X \in T_{b}(B)$; we thus have $b\left(\pi_{B^{*}}(X)\right)$ $=\sum_{\lambda=1}^{n+v} \Omega^{\lambda}(X) \boldsymbol{e}_{\lambda}$ in terms of the canonical basis $\left(\boldsymbol{e}_{\lambda}\right)$ of $\boldsymbol{R}^{n+\nu}$. The vector $X$ is tangent to the fiber of $B$ if and only if all $\Omega^{\lambda}(X)$ vanish.

Proposition 1.1. A section $s: M \rightarrow B$ comes from an isometric immersion $f=M \rightarrow \boldsymbol{R}^{n+v}$ so that one has $s=f^{B}$ if and only if the pullbacks $s^{*} \Omega^{\lambda}, 1 \leqq \lambda \leqq n+\nu$, are all exact (viz. closed and cohomologous to zero). When the above hypothesis is satisfied, $f^{B}$ determines $f$ uniquely up to the translations of $\boldsymbol{R}^{n+v}$.

Proof. Assume $s^{*} \Omega^{\lambda}$ are exact so that there exists a function $f^{\lambda}: M \rightarrow \boldsymbol{R}$ satisfying $s^{*} \Omega^{\lambda}=d f^{\lambda}$. Then a map $f: M \rightarrow R^{n+v}$ is defined by $f(x)=\left(f^{\lambda}(x)\right)_{1 \leqq \lambda \leqq n+v}$ $=\sum f^{\lambda}(x) e_{\lambda}$. We claim that $f$ is an isometric immersion. For any tangent vector 
$X \in T_{x}(M)$ to $M$ we have $s_{*} X \in T_{s(x)}(B)$ and $f_{*}(X)=\left(d f^{\lambda}(X)\right)=\left(s^{*} \Omega^{\lambda}(X)\right)$ $=\left(\Omega^{\lambda}\left(s_{*} X\right)\right)=s(x) \circ \pi_{B^{*}}\left(s_{*}(X)\right)=s(x)(X)$ since $\pi_{B} \circ s$ is the identity map of $M$. Thus $f_{*} \mid T_{x}(M)=s(x)$ and this must be a linear isometry; $f_{*} \mid T_{x}(M)=f^{B}(x)$. Hence $f$ is an isometric immersion. Conversely, assume $s=f^{B}$ for some isometric immersion $f: M \rightarrow \boldsymbol{R}^{n+\nu}$. Let $f^{\lambda}$ be the $\lambda$ th component of $f ; f(x)=\sum f^{\lambda}(x) \boldsymbol{e}_{\lambda}$. Then we have $\left(s^{*} \Omega^{\lambda}(X)\right)=\left(\Omega^{\lambda}\left(s_{*} X\right)\right)=s(x) \circ \pi_{B^{*}}\left(s_{*}(X)\right)=s(x)(X)=f^{B}(x)(X)=f_{*}(X)=\left(d f^{\lambda}(X)\right)$ for each $X \in T_{x}(M)$. Thus $s^{*} \Omega^{\lambda}=d f^{\lambda}$ and $s^{*} \Omega^{\lambda}$ is exact. This $f^{\lambda}$ is unique up to a constant. Hence $f$ is unique up to a translation of $\boldsymbol{R}^{n+v}$. Q.E.D.

Analogous forms $\omega^{i}, 1 \leqq i \leqq n$, are defined on the orthogonal frame bundle $P=\left\{b^{-1} \mid b \in B_{0}\right\} ; \sum_{i=1}^{n} \omega^{i}(X) e_{i}=b\left(\pi_{P}(X)\right)$, or $b^{-1}\left(\sum \omega^{i}(X) e_{i}\right)=\pi_{P}(X)$, or equivalently, if $\iota$ denotes the map $P \rightarrow B$ defined by $\iota\left(b^{-1}\right)=b$, then $\omega^{i}=\iota^{*} \Omega^{i}$ where $\left(\Omega^{i}\right)$ is the canonical form of $B_{0} .\left(\omega^{i}\right)$ is called the canonical form of $P$. (We use $P$ instead of $B_{0}$ just to conform to custom.)

2. A generating system of differential forms. Our convention on the ranges of the indices is: $1 \leqq \kappa, \lambda, \mu, \ldots \leqq n+\nu ; 1 \leqq i, j, k, \ldots \leqq n ; n+1 \leqq \alpha, \beta, \gamma, \ldots \leqq n+\nu$.

We will define particular forms on $P \times \mathrm{SO}(n+\nu)$ to express other forms with them. We begin by fixing notations for the Maurer-Cartan form $\Omega=\left(\Omega_{\mu}^{\lambda}\right)$ of SO $(n+\nu)$. SO $(n+\nu)$ consists of the unimodular orthogonal $(n+\nu) \times(n+\nu)-$ matrices $V=\left(V_{\mu}^{\lambda}\right)$, i.e.

$$
\sum_{\kappa=1}^{n+\nu} V_{\lambda}^{\kappa} V_{\mu}^{x}=\delta_{\lambda \mu}, \quad 1 \leqq \lambda, \mu \leqq n+\nu,
$$

and det $\left(V_{\mu}^{\lambda}\right)=1$. We understand that $\left(V_{\mu}^{\lambda}\right)$ is a linear transformation of $\boldsymbol{R}^{n+v}$ which sends $e_{\lambda}$ to $\sum_{\mu=1}^{n+v} V_{\lambda}^{\mu} e_{\mu}$. Each $V_{\mu}^{\lambda}$ is a well-defined function on $\mathrm{SO}(n+\nu)$. If we put

$$
\Omega_{\mu}^{\lambda}=\sum_{\kappa=1}^{n+v} V_{\lambda}^{x} d V_{\mu}^{x},
$$

or, in matrix expression, $\Omega={ }^{t} V d V$, we obtain the Maurer-Cartan form on $\mathrm{SO}(n+\nu)$. The $\Omega_{\mu}^{\lambda}$ are one-forms on SO $(n+v)$ which are left-invariant in the sense that we have ${ }^{t}(A V) d(A V)={ }^{t} V^{t} A A d V={ }^{t} V d V$ for any fixed $A \in \mathrm{SO}(n+\nu)$, while the right transform ${ }^{t}(V A) d(V A)={ }^{t} A\left({ }^{t} V d V\right) A={ }^{t} A \Omega A$ is not necessarily $\Omega$. We note that these forms $\Omega_{\mu}^{\lambda}$ span the cotangent space to SO $(n+\nu)$ at each point and that we have

$$
\Omega_{\lambda}^{\mu}=-\Omega_{\mu}^{\lambda}, \quad 1 \leqq \lambda, \mu \leqq n+\nu,
$$

which we readily see from (2.1) and (2.2). $\left(\Omega_{\mu}^{\lambda}\right)_{1 \leqq \lambda<\mu \leqq n+v}$ gives a basis of the cotangent space to $\mathrm{SO}(n+\nu)$ at each point. If $A \in \mathrm{SO}(n+\nu)$ and $Y$ is a skewsymmetric $(n+\nu) \times(n+\nu)$-matrix (i.e. a member of the Lie algebra of SO $(n+\nu))$, then $A Y$ is thought of as a tangent vector to $\mathrm{SO}(n+\nu)$ at $A$ and we have $\Omega(A Y)$ $={ }^{t} A A Y=Y$. 
Next we pull back these forms $\Omega_{\mu}^{\lambda}, 1 \leqq \lambda, \mu \leqq n+\nu$, to $P \times \operatorname{SO}(n+\nu)$ from SO $(n+\nu)$ by the natural projection $P \times \mathrm{SO}(n+\nu) \rightarrow \mathrm{SO}(n+\nu)$, and denote the resulting forms on $P \times \operatorname{SO}(n+\nu)$ by the same symbols $\Omega_{\mu}^{\lambda}$.

Furthermore, we pull back the canonical form $\left(\omega^{i}\right)_{1 \leqq i \leqq n}$ and the Riemannian connection form $\left(\omega_{j}^{i}\right)_{1 \leqq i, j \leqq n}$ from $P$ to $P \times S O(n+\nu)$ by the natural projection of $P \times \mathrm{SO}(n+\nu)$ onto $P$. The unique existence of $\left(\omega_{j}^{i}\right)$ is well known. The connection $\left(\omega_{j}^{i}\right)$ is characterized by the following conditions: (1) $\omega_{i}^{j}=-\omega_{j}^{i},(2)\left(\omega_{j}^{i}(p Y)\right)=Y$ for $p \in P$, and any member $Y$ of the Lie algebra of SO $(n),(3)\left(\omega_{j}^{i}(X g)\right)={ }^{t} g\left(\omega_{j}^{i}(X)\right) g$ for $X \in T(P)$ and $g \in \mathrm{SO}(n)$, and (4) $\left(\omega_{j}^{i}\right)$ is torsion free, meaning (2.5) below. The forms so obtained on $P \times \mathrm{SO}(n+\nu)$ will be denoted by the same symbols $\omega^{i}$ and $\omega_{j}^{i}$.

The forms $\Omega_{\mu}^{\lambda}, \omega_{j}^{i}, \omega^{k}$ span the cotangent space to $P \times S O(n+\nu)$ at each point and, if we impose the restrictions $\lambda<\mu$ and $i<j$, they become a basis. Thus the differential forms on $P \times \mathrm{SO}(n+\nu)$ form the exterior algebra generated by $\Omega_{\mu}^{\lambda}, \omega_{j}^{i}, \omega^{k}$, $1 \leqq \lambda<\mu \leqq n+\nu, 1 \leqq i<j \leqq n, 1 \leqq k \leqq n$, over the ring of differentiable functions on $P \times \mathrm{SO}(n+v)$.

In particular, the canonical form $\left(\Omega^{\lambda}\right)$ of $B$, pulled back to $P \times \mathrm{SO}(n+\nu)$ via $P \times V_{n+v, n}$ by the projection: $P \times \mathrm{SO}(n+\nu) \rightarrow P \times V_{n+v, n} \stackrel{\pi}{\longrightarrow} B$, is expressed as

LemMA 2.1. $\Omega^{\lambda}=\sum_{i=1}^{n} V_{i}^{\lambda} \omega^{i}$, or equivalently $\omega^{i}=\sum_{\lambda=1}^{n+v} V_{i}^{\lambda} \Omega^{\lambda}$ on $P \times \mathrm{SO}(n+\nu)$.

This will be proved shortly later.

The exterior derivatives of those generators above are given by the following "structure equations":

and

$$
\begin{aligned}
d \Omega_{\mu}^{\lambda} & =\sum_{\kappa=1}^{n+v} \Omega_{\lambda}^{\kappa} \wedge \Omega_{\mu}^{\kappa}, \\
d \omega^{i} & =\sum_{j=1}^{n} \omega^{j} \wedge \omega_{j}^{i},
\end{aligned}
$$

where $K=\left(K_{j k h}^{i}\right)$ is the curvature of the Riemannian connection whose components $K_{j k h}^{i}$ are functions with convention $K_{j h k}^{i}=-K_{j h k}^{i}$. (2.4) follows from (2.2) and (2.1). (2.5) was contained in the definition of $\left(\omega_{j}^{i}\right)$. (2.6) is well known.

To prove Lemma 2.1, it is sufficient to show that the formulas in Lemma 2.1 make sense and are valid on $P \times V_{n+v, n}$. They will make sense if the functions $V_{i}^{\lambda}, 1 \leqq i \leqq n, 1 \leqq \lambda \leqq n+\nu$, are well defined on $V_{n+v, n}$ or $P \times V_{n+v, n}$ since other forms are clearly well defined on $P \times V_{n+v, n} . V_{n+v, n}$ was identified with $\mathrm{SO}(n+v) / \mathrm{SO}(v)$ in $\S 1$. If $V=\left(V_{\mu}^{\lambda}\right) \in \mathrm{SO}(n+\nu)$ is mapped to $v \in V_{n+v, n}$ by the natural projection:

$$
\mathrm{SO}(n+\nu) \rightarrow \mathrm{SO}(n+\nu) / \mathrm{SO}(\nu)=V_{n+v, n}, v
$$

is given by the submatrix $\left(V_{i}^{\lambda}\right)_{1 \leqq i \leqq n, 1 \leqq \lambda \leqq n+v}$; in fact, $V$ sends each basis vector $\boldsymbol{e}_{\mu}, 1 \leqq \mu \leqq n+\nu$, to $\sum_{\lambda=1}^{n+v} V_{\mu}^{\lambda} e_{\lambda}$ and in particular, the first $n$ vectors $e_{i}, 1 \leqq i \leqq n$, are sent to $\sum V_{i}^{\lambda} e_{\lambda}$ or $v\left(e_{i}\right)=\sum V_{i}^{\lambda} e_{\lambda}$. Now we will prove the formulas in Lemma 2.1 
on $P \times V_{n+v, n}$. Let $(X, Y)$ be an arbitrary tangent vector to $P \times V_{n+v, n}$ at a point $(p, v) ; X \in T_{p}(P), \quad Y \in T_{v}\left(V_{n+v, n}\right)$. Put $b=\pi(p, v)$. Then the definition of $\Omega^{\lambda}$ reads $\sum_{\lambda=1}^{n+\nu} \Omega^{\lambda}((X, Y)) e_{\lambda}=b\left(\pi_{B^{*}}(X, Y)\right)$. We have $\pi_{B^{*}}\left(\pi_{*}(X, Y)\right)=\pi_{B^{*}}\left(\pi_{*}(X, 0)\right)=\pi_{P^{*}}(X)$ since $(X, Y)=(X, 0)+(0, Y)$ and $\pi_{*}(0, Y)$ is tangent to the fiber of $B$ through $b$. On the other hand, we recall (see the end of $\S 1$ ) that the canonical form $\left(\omega^{i}\right)$ of $P$ satisfies $p\left(\sum_{i=1}^{n} \omega^{i}(X) e_{i}\right)=\pi_{P^{*}}(X)$ or $\sum \omega^{i}(X) e_{i}=p^{-1}\left(\pi_{P^{*}}(X)\right)$. Hence, it follows from the relation $b=\pi(p, v)=v \circ p^{-1}$ that we have

$$
\begin{aligned}
b\left(\pi_{B^{\bullet}}\left(\pi_{*}(X, Y)\right)\right) & =b\left(\pi_{P^{*}}(X)\right)=\left(v \circ p^{-1}\right)\left(\pi_{P^{*}}(X)\right)=v\left(\sum \omega^{i}(X) \boldsymbol{e}_{i}\right) \\
& =\sum \omega^{i}(X) v\left(\boldsymbol{e}_{i}\right)=\sum_{i} \omega^{i}(X) \sum_{\lambda=1}^{n+1} V_{i}^{\lambda} e_{\lambda} .
\end{aligned}
$$

Thus, we conclude $\sum \Omega^{\lambda}((X, Y)) e_{\lambda}=\sum V_{i}^{\lambda} \omega^{i}(X) e_{\lambda}$ and $\Omega^{\lambda}=\sum V_{i}^{\lambda} \omega^{i}$, since $\omega^{i}(X)$ should be understood as $\omega^{i}((X, Y))$. That the formula $\Omega^{\lambda}=\sum V_{i}^{\lambda} \omega^{i}$ is equivalent to $\omega^{i}=\sum_{\lambda} V_{i}^{\lambda} \Omega^{\lambda}$ follows from (2.1); indeed we have $\sum_{\lambda} V_{i}^{\lambda} \Omega^{\lambda}=\sum_{\lambda, j} V_{i}^{\lambda} V_{j}^{\lambda} \omega^{i}=\sum_{j} \delta_{i j} \omega^{j}$ $=\omega^{i}$. We have thus proved Lemma 2.1 together with the following modification:

Lemma $2.1^{\prime}$. On $P \times V_{n+v, n}$, the functions $V_{i}^{\lambda}, 1 \leqq \lambda \leqq n+v, 1 \leqq i \leqq n$, are well defined, and one has

$$
\Omega^{\lambda}=\sum_{i} V_{i}^{\lambda} \omega^{i} \text { and } \omega^{i}=\sum_{\lambda} V_{i}^{\lambda} \Omega^{\lambda}
$$

We have constructed differential forms on $P \times V_{n+v, n}$ to help us define certain significant forms on $B$. More precisely, given a certain form $\theta$ on $P \times \mathrm{SO}(n+\nu)$, we want to have a form $\theta_{B}$ on $B$ whose pull back to $P \times \mathrm{SO}(n+\nu)$ is $\theta$. When this is the case we will say that we can $\operatorname{dump} \theta$ to $B$ obtaining $\theta_{B}$. First, we note that, with the projection: $P \times \mathrm{SO}(n+\nu) \rightarrow P \times V_{n+v, n}, P \times \mathrm{SO}(n+v)$ is a principal SO (v)-bundle over $P \times V_{n+v, n}$ which in turn is a principal SO (n)-bundle over $B$ with the projection $\pi$, and finally $P \times \mathrm{SO}(n+v)$ is also thought of as a principal $\mathrm{SO}(n) \times \mathrm{SO}(v)$ bundle over $B$ with the projection $P \times \mathrm{SO}(n+\nu) \rightarrow P \times V_{n+v, n} \stackrel{\pi}{\longrightarrow} B$, as will be explained later.

Thus, the dumping will be done based on the following principle. Let $P$ be an arbitrary differentiable principal bundle which may be different from the frame bundle of $M$ in the rest of this paragraph. Let $M, \pi$ and $G$ denote the base manifold, the projection and the structure group respectively of $P$. If a differential form $\theta$ on $P$ is the pull back $\pi^{*} \theta_{M}$ of a form $\theta_{M}$, then (1) $\theta$ is $G$-invariant and (2) $\theta$ is transversal to the fibers in the sense that we have $\iota(Y) \theta=0$ for any tangent vector $Y$ to the fibers of $P$ where $\iota(Y) \theta$ means the inner product of $Y$ with $\theta ; \iota(Y) \theta\left(Y_{1}, Y_{2}, \ldots, Y_{r-1}\right)$ $=\theta\left(Y, Y_{1}, Y_{2}, \ldots, Y_{r-1}\right), r=\operatorname{deg} \theta$. (To see (1), let $G$ act on $M$ trivially. Then $\pi$ is equivariant. (2) follows simply from $\pi_{*}(Y)=0$.) Conversely, if a form $\theta$ on $P$ satisfies the conditions (1) and (2) then we can dump $\theta$ to $M$. In fact, since $\pi$ is a submersion (i.e. its differential $\pi_{*}: T_{p}(P) \rightarrow T_{\pi(p)}(M)$ is surjective if it is restricted to each tangent space $\left.T_{p}(P)\right)$, there exist $X_{1}^{\prime}, \ldots, X_{r}^{\prime} \in T_{p}(P)$ for any $X_{1}, \ldots, X_{r}$ $\in T_{x}(M)$ such that $\pi_{*}\left(X_{q}^{\prime}\right)=X_{q}, 1 \leqq q \leqq r$, where $p$ is a point in $\pi^{-1}(x), \theta\left(X_{1}^{\prime}, \ldots, X_{r}^{\prime}\right)$ is determined by $X_{1}, \ldots, X_{r}$ only and is independent of the choice of $X_{1}^{\prime}, \ldots, X_{r}^{\prime}$ as 
above by virtue of both (1) and (2). Although there would be other methods (e.g., to use a connection on $P$ ) to construct a form on $M$ from a given form $\theta$ on $M$, the dumping, has the advantage of the relation $d \theta=d \pi^{*} \theta_{M}=\pi^{*} d \theta_{M}$; this implies that we have $d \theta=0$ if and only if $d \theta_{M}=0$. (For the proof of the "only if" part, use the fact that $\pi$ is a submersion.)

Hereafter $P$ will denote the orthonormal frame bundle of our oriented Riemannian manifold $M$. We will apply the principle above to forms on our principal bundles $P \times \mathrm{SO}(n+\nu)$, etc. First we recall that every form $\theta$ on $P \times \mathrm{SO}(n+\nu)$ can be uniquely expressed with $\omega^{i}, \omega_{j}^{i}$ and $\Omega_{\mu}^{\lambda}$ along with the coefficient functions. Regarding $P \times \mathrm{SO}(n+\nu)$ as an SO (v)-bundle over $P \times V_{n+v, n}, \theta$ satisfies (1), i.e., $\theta$ is SO $(\nu)$-invariant if the coefficient functions for $\theta$ are constant and the expression of $\theta$ remains unchanged under the transformation: $\Omega=\left(\Omega_{\mu}^{\lambda}\right) \mapsto{ }^{t} a \Omega a$ for any $a \in \mathrm{SO}(\nu)$ or the substitutions of $\sum_{\gamma=n+1}^{n+v} \Omega_{\gamma}^{i} a_{\alpha}^{\gamma}, \quad 1 \leqq i \leqq n<\alpha \leqq n+\nu$, for $\Omega_{\alpha}^{i}$ and of $\sum_{\gamma, \delta=1}^{n+v} a_{\alpha}^{\delta} \Omega_{\alpha}^{\delta} a_{\beta}^{\alpha}$ for $\Omega_{\beta}^{\alpha}, n<\alpha, \beta \leqq n+\nu$, where $\left(a_{\beta}^{\alpha}\right)$ is an arbitrary member of SO $(\nu)$ identified with $\left\{1_{n}\right\} \times \mathrm{SO}(\nu) \subset \mathrm{SO}(n+\nu) . \theta$ is moreover transversal to the fibers if the expression of $\theta$ does not contain $\Omega_{\beta}^{\alpha}, n<\alpha, \beta \leqq n+\nu$, since the tangent vectors $Y$ on $(p, a) Y$ to the fibers are characterized by the vanishing of the inner products of $Y$ with the other forms $\omega^{i}$, etc. (and $\left(\Omega_{\beta}^{\alpha}(Y)\right)=Y$ if $Y$ is interpreted as a skew-symmetric matrix). For instance, we can dump $\Omega_{j}^{i}, 1 \leqq i, j \leqq n$, to $P \times V_{n+v, n}$; although one may note that this fact follows also from Lemma 2.1' and the definition (2.2). Hence, it makes sense to consider a linear differential system $\Omega_{j}^{i}-\omega_{j}^{i}=0,1 \leqq i, j \leqq n$, on $P \times V_{n+v, n}$, which will appear in later sections.

Furthermore, we wish to dump $\theta$ on $P \times \mathrm{SO}(n+\nu)$ to $B$. If this is possible, we can dump it to $P \times V_{n+v, n}$. In any case, $P \times \mathrm{SO}(n+v)$ is a principle $\mathrm{SO}(n) \times \mathrm{SO}(\nu)$ bundle over $B$. Each $(g, h) \in \mathrm{SO}(n) \times \mathrm{SO}(\nu)$ sends $(p, a)$ to $(p g, a(g \times h))$ where $g \times h$ is considered as a member of SO $(n+\nu)$ which sends $e_{\lambda}$ to $g\left(e_{\lambda}\right)$ or $h\left(e_{\lambda}\right)$ $=\left(1_{n} \times h\right)\left(e_{\lambda}\right)$ depending on whether $\lambda \leqq n$ or $\lambda>n$. A form $\theta$ on $P \times \operatorname{SO}(n+\nu)$ can be dumped to $B$ if $\theta$ is SO $(n) \times \mathrm{SO}(\nu)$-invariant and $\theta$ is transversal to the fibers. To see when the second condition is satisfied, we will find the relation between the tangent vectors $Y$ to the fibers and the values of the basic forms $\Omega_{\mu}^{\lambda}$, etc. for $Y$. A member of the Lie algebra of SO $(n) \times \mathrm{SO}(\nu)$ is a skew-symmetric $(n+v) \times(n+v)$ matrix, which we denote by the pair $\left(Y_{1}, Y_{2}\right)$ of the skew-symmetric matrices $Y_{1}$ of size $n$ and $Y_{2}$ of size $\nu$ such that $Y_{1}\left(e_{\alpha}\right)=0, n<\alpha \leqq n+\nu$, and $Y_{2}\left(e_{i}\right)=0,1 \leqq i \leqq n$. This $\left(Y_{1}, Y_{2}\right)$ gives rise to a vector field $\tilde{Y}$ on $P \times \operatorname{SO}(n+v)$ whose value at $(p, a)$ is written as $\left(p Y_{1}, a Y_{1}+a Y_{2}\right)$ in a natural way. For a fixed $(p, a)$, this map: $\left(Y_{1}, Y_{2}\right) \mapsto\left(p Y_{1}, a Y_{1}+a Y_{2}\right)$ is an isomorphism of the vector space of the pairs $\left(Y_{1}, Y_{2}\right)$ onto the tangent space to the fiber through $(p, a)$ at $(p, a)$. We have the matrix

$$
\begin{aligned}
\left(\Omega_{j}^{i}\left(\left(p Y_{1}, a Y_{1}+a Y_{2}\right)\right)\right) & =\left(\Omega_{j}^{i}\left(a Y_{1}\right)\right)=Y_{1}, \\
\Omega_{\beta}^{\alpha}\left(\left(p Y_{1}, a Y_{1}+a Y_{2}\right)\right) & =\left(\Omega_{\beta}^{\alpha}\left(Y_{2}\right)\right)=Y_{2}, \\
\left(\omega_{j}^{i}\left(\left(p Y_{1}, a Y_{1}+a Y_{2}\right)\right)\right) & =\left(\omega_{j}^{i}\left(p Y_{1}\right)\right)=Y_{1} \text { for } 1 \leqq i, j \leqq n, n<\alpha, \beta \leqq n+\nu,
\end{aligned}
$$


and the inner products of $\tilde{Y}(p, a)=\left(p Y_{1}, a Y_{1}+a Y_{2}\right)$ with the other basic forms $\Omega_{\beta}^{i}=-\Omega_{i}^{\beta}, \omega^{i}, 1 \leqq i \leqq n<\beta<n+\nu$, are all zero. We have thus proved:

Lemma 2.2. A form $\theta$ on $P \times \mathrm{SO}(n+\nu)$ can be dumped to $B$ (i.e. $\theta$ is a pullback of $a$ form on $B$ by the projection) if and only if $\theta$ is $\mathrm{SO}(n) \times \mathrm{SO}(\nu)$-invariant and $\theta$ does not contain either $\omega_{j}^{i}+\Omega_{j}^{i}$ or $\Omega_{\beta}^{\alpha}$ so that $\theta$ can be expressed only by $\Omega_{\beta}^{i}$, $\omega_{j}^{i}-\Omega_{j}^{i}$, and $\omega^{i}, 1 \leqq i, j \leqq n<\alpha, \beta \leqq n+\nu$.

3. Prolongations of the differential system for the isometric immersions. We keep the notations of preceding sections in this and subsequent sections.

Definition. A subspace $H$ of the tangent space $T_{b}(B)$ at a point $b$ is called $a$ holonomic horizontal plane if and only if

(3.1) The restriction $\pi_{B^{*}} \mid H$ is an isomorphism onto $T_{x}(M), x=\pi_{B}(b)$, and

(3.2) each $d \Omega^{\lambda}=0$ on $H, 1 \leqq \lambda \leqq n+\nu$, where $\pi_{B}$ is the projection: $B \rightarrow M$. The requirement (3.2) means $d \Omega^{\lambda}\left(X_{1}, X_{2}\right)=0$ for $X_{1}, X_{2} \in H$. We have $\operatorname{dim} H=n$ = $\operatorname{dim} M$ by (3.1). A holonomic horizontal plane $H$ is "an integral element" of "the differential system" given by $d \Omega^{\lambda}=0$ with $M$ as the space of "the independent variables," in the terminologies of the Cartan-Kuranishi theory. The purpose of this section is to state and prove several propositions and lemmas to be used in $\S 5$.

Proposition 3.1. The collection $\mathscr{H}=\{\boldsymbol{H}\}$ of all the holonomic horizontal planes $\boldsymbol{H}$ is naturally a (nonempty) differentiable subbundle of the Grassmann bundle consisting of all the n-dimensional subspaces of the tangent spaces to $B$.

The geometric meaning of the differential system $\mathscr{H}$ is given by

Proposition 3.2. Locally, the maximal dimensional (i.e. n-dimensional) integral manifolds of $\mathscr{H}$ are in a one-to-one correspondence with the isometric immersions of $M$ onto $\boldsymbol{R}^{n+v}$ modulo the translations of $\boldsymbol{R}^{n+v}$.

In other words $\mathscr{H}$ is "a prolongation" of the differential equation for the isometric immersions of $M$ into $\boldsymbol{R}^{n+\nu}$.

By means of the projection $\pi: P \times V_{n+v, n} \rightarrow B$, we pull $H$ back to $\pi^{*} \mathscr{H}$. Thus, given a point $(p, v) \in P \times V_{n+v, n}$ let $\left(\pi^{*} \mathscr{H}\right)_{(p, v)}$ denote the set of all the complete inverse images $\left\{\pi_{*}^{-1}(H) \mid H \in \mathscr{H}, H \subset T_{\pi(p, v)}(B)\right\}$ where $\pi_{*}$ is understood as its restriction to $T_{(p, v)}\left(P \times V_{n+v, n}\right)$. Let $\pi^{*} \mathscr{H}$ denote the union of $\left(\pi^{*} \mathscr{H}\right)_{(p, v)}$ for all $(p, v)$ $\in P \times V_{n+v, n} . \pi^{*} \mathscr{H}$ will turn out to be a differentiable fiber bundle over $P \times V_{n+v, n}$. We will write $\pi^{*} H$ for $\pi_{*}^{-1}(H)$.

LEMMA 3.3. $\omega_{j}^{i}=\Omega_{j}^{i}, 1 \leqq i, j \leqq n$, on each $\pi^{*} H \in \pi^{*} \mathscr{H}$.

LeMMA 3.4. Each $\pi^{*} H \in \pi^{*} \mathscr{H}$ contains a unique subspace $H_{0}$ such that (1) $\omega_{j}^{i}=\Omega_{j}^{i}=0$ on $H_{0}$ and (2) $\pi_{*}: H_{0} \rightarrow H$ is an isomorphism.

We begin the demonstrations by proving:

$$
\sum_{\lambda=1}^{n+v} V_{j}^{\lambda} d \Omega^{\lambda}=\sum_{i=1}^{n}\left(\Omega_{i}^{j}-\omega_{i}^{j}\right) \wedge \omega^{i}, \quad 1 \leqq j \leqq n,
$$

on $P \times V_{n+v, n}$. 
From $\Omega^{\lambda}=\sum V_{i} \omega^{i}$ (Lemma 2.1'), we obtain $d \Omega^{\lambda}=\sum\left(d V_{i}^{\lambda} \wedge \omega^{i}+V_{i}^{\lambda} d \omega^{i}\right)$. It follows from (2.5) and (2.2) that $\sum_{\lambda} V_{j}^{\lambda} d \Omega^{\lambda}=\sum_{i} \Omega_{i}^{j} \wedge \omega^{i}+d \omega^{j}=\sum_{i}\left(\Omega_{i}^{j} \wedge \omega^{i}+\omega^{i} \wedge \omega_{i}^{j}\right)$ $=\sum\left(\Omega_{i}^{j}-\omega_{i}^{j}\right) \wedge \omega^{i}$, and $(3.3)$ is proved.

Since the matrix $\left(V_{i}^{\lambda}\right)_{1 \leqq i \leqq n, 1 \leqq \lambda \leqq n+v}$ has rank $n$, we have $\sum_{\lambda} V_{i}^{\lambda} d \Omega^{\lambda}=0,1 \leqq i \leqq n$, on a subspace, say $U$, of $T_{(p, v)}\left(P \times V_{n+v, n}\right)$ if and only if we have $d \Omega^{\lambda}=0,1 \leqq \lambda \leqq n+\nu$, on $U$. Hence, in view of (3.3), we have $d \Omega^{\lambda}=0$ on $U$ provided $\Omega_{i}^{j}-\omega_{i}^{j}=0$ on $U$. Let $U_{(p, v)}$ be the subspace of $T_{(p, v)}\left(P \times V_{n+v, n}\right)$ defined by the linear equations $\Omega_{i}^{j}-\omega_{i}^{j}=0$, $1 \leqq i, j \leqq n$. We have $d \Omega^{\lambda}=0,1 \leqq \lambda \leqq n+\nu$, on $U_{(p, v)}$ by the above. Let $H^{\prime}$ be an $n-$ dimensional subspace (provided it exists) of $U_{(P, v)}$ on which

$$
\omega^{1}, \omega^{2}, \ldots, \omega^{n} \text { are linearly independent. }
$$

Such an $H^{\prime}$ does indeed exist since the forms $\omega^{i}$ are linearly independent of $\Omega_{j}^{i}-\omega_{j}^{i}, 1 \leqq i, j \leqq n$, on $T_{(p, v)}\left(P \times V_{n+v, n}\right)$ and hence on $U_{(p, v)}$. We have $d \Omega^{\lambda}=0$ on $H^{\prime} \subseteq U_{(p, v)}$. Again by Lemma $2.1^{\prime}$, there exist $n$ one-forms among $\Omega^{\lambda}, 1 \leqq \lambda \leqq n+\nu$, which are linearly independent on $H^{\prime}$. This fact is also true for $\pi_{*}\left(H^{\prime}\right)$, and so, by the definition of $\Omega^{\lambda}, \pi_{*}\left(H^{\prime}\right)$ satisfies (3.1). $\pi_{*}\left(H^{\prime}\right)$ satisfies (3.2) too. Therefore, $\pi_{*}\left(H^{\prime}\right)$ is a holonomic horizontal plane. In particular, $\mathscr{H}$ is not empty. Conversely, given an $H \in \mathscr{H}$, we will show that $\omega_{i}^{j}=\Omega_{i}^{j}$ on $\pi^{*} H$ and thereby establish Lemma 3.3. On this space $\pi^{*} H$ we have (3.4) and $d \Omega^{\lambda}=0$ by (3.1) and (3.2) respectively. Thus $\pi^{*} H$ satisfies the hypothesis for $U$ in the Lemma 3.5 below, and Lemma 3.3 follows.

LEMMA 3.5. A subspace $U$ of $T_{(p, v)}\left(P \times V_{n+v, n}\right)$ is contained in $U_{(p, v)}$, i.e. $\omega_{i}^{j}=\Omega_{i}^{j}$ on $U$ if we have (3.4) for $U$ and $d \Omega^{\lambda}=0,1 \leqq \lambda \leqq n+\nu$, on $U$.

Proof. (This is equivalent to the uniqueness of the Riemannian connection, or rather to the fact that the linear group $\mathrm{SO}(n)$ is of the order one.) By (3.3) and $d \Omega^{\lambda}=0$, we have on $U$

$$
\sum_{i}\left(\Omega_{i}^{j}-\omega_{i}^{j}\right) \wedge \omega^{i}=0 .
$$

Therefore, $\Omega_{i}^{j}-\omega_{i}^{j}$ is a linear combination $\sum_{k=1}^{n} A_{i k}^{j} \omega^{k}$ of $\omega^{1}, \omega^{2}, \ldots, \omega^{n}$ by (3.4) and the so-called Cartan lemma, where $A_{j k}^{i} \in R$. Substituting $\sum A_{j k}^{i} \omega^{k}$ for $\Omega_{i}^{j}-\omega_{i}^{j}$ in (3.6), we see that $A_{i k}^{j}$ is symmetric in the indices $i$ and $k ; A_{k i}^{j}=A_{i k}^{j}$. On the other hand we have $A_{j k}^{i}=-A_{i k}^{j}$ since $\Omega_{i}^{j}-\omega_{i}^{j}$ is skew-symmetric in $i$ and $j$. From these two properties of $\left(A_{j k}^{i}\right)$ we infer that $A_{j k}^{i}=A_{k j}^{i}=-A_{i j}^{k}=-A_{j i}^{k}=A_{k i}^{j}=A_{i k}^{j}=-A_{j k}^{i}$, whence $A_{j k}^{i}=0$ or $\Omega_{i}^{j}-\omega_{i}^{j}=0$ and Lemma 2.5 is proved.

Without going into the details of the proof, we next show that $\mathscr{H}$ and $\pi^{*} \mathscr{H}$ are differentiable fibre bundles over $B$ and $P \times V_{n+v, n}$ respectively. In fact, the holonomic horizontal planes $H$ at an arbitrarily fixed point $b \in B$ form a subset $\mathscr{H}_{b}$ of $\mathscr{H}$ which is in a one-to-one correspondence with $\left(\pi^{*} \mathscr{H}\right)_{(p, v)}$ for a fixed $(p, v) \in B \times V_{n+v, n}$ with $\pi(p, v)=b$. And a member $\pi^{*} H$ of $\left(\pi^{*} \mathscr{H}\right)_{(p, v)}$ is characterized as a subspace (Lemma 3.3) of $U_{(p, q)}$ which is the direct sum of an $n$-dimensional subspace $H^{\prime}$ 
satisfying (3.4) and the tangent space, denoted by $\left(\operatorname{Ker} \pi_{*}\right)_{(p, v)}$, to the fiber through $(p, v)$ at $(p, v)$ of the principal bundle $P \times V_{n+v, n}$ over $B ; \pi^{*} H=H^{\prime} \oplus\left(\operatorname{Ker} \pi_{*}\right)_{(p, v) \text {. }}$ If we fix a subspace $U_{(p, b)}^{\prime}$ of $U_{(p, q)}$ which is complementary to $\left(\operatorname{Ker} \pi_{*}\right)_{(p, v)}$, then we have (3.4) and $d \Omega^{\lambda}=0$ since we have these on $U_{(p, q)}$ and $\omega^{i}$ are zero on $\left(\operatorname{Ker} \pi_{*}\right)_{(p, v) \text {. }}$ The set of all $n$-dimensional subspaces $H^{\prime}$ contained in $U_{(p, q)}^{\prime}$ and satisfying (3.4) is in a one-to-one correspondence with $\left(\pi^{*} \mathscr{H}\right)_{(p, v)}$ and it is open in the Grassmann manifold of all $n$-dimensional subspaces of $U_{(p, v)}^{\prime}$. We want to choose $U_{(p, v)}^{\prime}$ for each $(p, v)$ in such a way that the union of all $U_{(p, v)}^{\prime},(p, v) \in P \times V_{n+v, n}$ naturally becomes a differentiable bundle over $P \times V_{n+v, n}$. Let $U_{(p, v)}^{\prime}$ be the subspace of $U_{(p, v)}$ which is given the linear equations $\omega_{j}^{i}=0,1 \leqq i, j \leqq n$. Then we have $U_{(p, v)}=U_{(p, v)}^{\prime} \oplus$ $\left(\operatorname{Ker} \pi_{*}\right)_{(p, v)}$ since we have $\left(\omega_{j}^{i}(p Y, v Y)\right)=Y$ for any tangent vector $(p Y, v Y)$ in $\left(\operatorname{Ker} \pi_{*}\right)_{(p, v)}$, where $Y$ is an arbitrary skew-symmetric $n \times n$ matrix. This incidentally proves Lemma 3.4; indeed, $H_{0}=\pi^{*} H \cap U_{(p, v)}^{\prime}$. Also we see from the arguments above that differentiable bundle structures are defined on $\pi^{*} \mathscr{H}$ and hence on $\mathscr{H}$. So Proposition 3.1 is proven. It remains to establish Proposition 3.2. Let $S$ be an integral manifold of $\mathscr{H}$ so that each tangent space to $S$ belongs to $\mathscr{H}$. By (3.1), $\pi_{B} \mid S$ is a local diffeomorphism. Let $f^{B}$ be the local diffeomorphism (defined on a small open set in $M)$ from $\pi_{B}(S)$ to $S$ which is the local inverse map of $\pi_{B} \mid S$. Then $f^{B^{*}} \Omega^{\lambda}$ is closed by (3.2). Hence locally $f^{B^{*}} \Omega^{\lambda}$ is exact. Thus, by Proposition 1.1, $f^{B}$ comes from a local isometric immersion $f$. Conversely, if $f$ is an isometric immersion of an open set of $M$ into $R^{n+v}$, the corresponding local section $f^{B}$ of $B$ gives rise to an integral manifold $=\operatorname{Image}\left(f^{B}\right)$ of $\mathscr{H}$, since its tangent spaces satisfy (3.1) (because of the section $f^{B}$ ) and (3.2) by Proposition 1.1.

REMARK 3.1. When $\nu=0$, it is not hard to see that $\mathscr{H}$ is a vector subbundle of the tangent bundle of $B=B_{0}$ and, moreover, it is a connection of $B_{0}$, which gives the Riemannian connection of $M$ when $\mathscr{H}$ is transplanted to $P$.

REMARK 3.2. For an isometric immersion $f: \boldsymbol{M} \rightarrow \boldsymbol{R}^{n+v}$ we obtain the map $f_{P}: P \rightarrow V_{n+v, n}$ such that $\pi\left(p, f_{P}(v)\right)=f^{B}\left(\pi_{P}(p)\right)$. It is seen that the tangent spaces to the graph of $f_{P}$ are contained in $U_{(p, v)}, v=f_{P}(p)$, and hence $\omega_{j}^{i}=f_{P}^{*} \Omega_{j}^{i}, 1 \leqq i, j \leqq n$. (Kobayashi [7]). This proves the existence of the Riemannian connection $\left(\omega_{j}^{i}\right)$ under the assumption of the existence of some $f$.

4. A regular homotopy invariant. For the sake of simplicity we will assume for the rest of the paper that $M$ is compact. In this section we will construct a regular homotopy invariant for the isometric immersions $f: M \rightarrow \boldsymbol{R}^{n+v}$ assuming that the codimension $\nu$ is even.

We first put $\Theta_{\beta}^{\alpha-n}=\sum_{i=1}^{n} \Omega_{\alpha}^{i} \wedge \Omega_{\beta}^{i}, n<\alpha, \beta \leqq n+\nu$. This is a form on $\operatorname{SO}(n+\nu)$. We pull it back to $P \times \mathrm{SO}(n+\nu)$. Then we put

$$
\Theta=\sum_{\tau} \operatorname{sgn}(\tau) \Theta_{\tau(2)}^{\tau(1)} \wedge \Theta_{\tau(4)}^{\tau(3)} \wedge \cdots \wedge \Theta_{\tau(v)}^{\tau(v-1)},
$$

where $\operatorname{sgn}(\tau)$ is the sign of the permutation $\tau$ of $\{1,2, \ldots, \nu\}$ and the summation ranges over all permutations. In this definition of $\Theta$, we have used the assumption 
that $\nu$ is even. We will show that we can dump $\Theta$ to $B$ to obtain a closed form $\Theta_{B}$. First $\Theta$ is invariant under the right action of $\left\{1_{n}\right\} \times$ SO $(\nu)$. This can be proven in various ways. For instance, since each $\Theta_{q}^{p}, 1 \leqq p, q \leqq \nu$, is of even degree, $\Theta$ can be thought of as a polynomial in the entries of $\Theta_{q}^{p}$ of the matrix $\left(\Theta_{q}^{p}\right)$. Now it is known (see Chern [1]) that $\Theta$ is essentially $\left(\operatorname{det}\left(\Theta_{q}^{p}\right)\right)^{1 / 2}$, and so the invariance follows. Next $\Theta$ is invariant under the right action of $\mathrm{SO}(n)$. Really each $\Theta_{\beta-n}^{\alpha-n}=\sum_{i=1}^{n} \Theta_{\alpha}^{i} \wedge \Theta_{\beta}^{i}$ is invariant, since $\mathrm{SO}(n)$ leaves invariant the polynomial $\sum_{i=1}^{n}\left(x^{i}\right)^{2} \cdot\left(g=\left(g_{j}^{i}\right) \in \mathrm{SO}(n)\right.$ sends $\Theta_{\alpha}^{i}$ to $\sum_{j=1}^{n} g_{j}^{i} \Theta_{\alpha}^{j}$.) It is obvious from Lemma 2.2 that $\Theta$ is transversal to the fibers of the principal bundle $P \times \mathrm{SO}(n+\nu)$ over $B$. Finally, we have to prove that $\Theta_{B}$ is closed, which is true if $\Theta$ is closed. Again there are various proofs. We will give two of them briefly. We regard $\Theta$ as a form on $\mathrm{SO}(n+\nu)$. Since $\Theta$ is $\mathrm{SO}(n)$ $\times \mathrm{SO}(\nu)$-invariant and $\Theta$ is transversal to the fibers of the principal bundle SO $(n+\nu)$ over the Grassmann manifold (by arguments similar to the proof of Lemma 2), $\Theta$ can be dumped to the Grassmann manifold to obtain a (left) SO $(n+v)$-invariant form. On the other hand, a Grassmann manifold is a compact symmetric space and any invariant form on such a space is closed by a well-known theorem of E. Cartan. Thus $\Theta$ is closed. A more elementary proof goes like this: $\Theta_{\beta-n}^{\alpha-n}=d \Omega_{\beta}^{\alpha}-\sum_{\gamma=n+1}^{n+\nu} \Omega_{\alpha}^{\gamma} \wedge \Omega_{\beta}^{\gamma}$ by (1.4). Hence, $d \Theta_{\beta-n}^{\alpha-n}=\sum\left(\left(d \Omega_{\alpha}^{\gamma}\right) \wedge \Omega_{\beta}^{\gamma}-\Omega_{\alpha}^{\gamma} \wedge d \Omega_{\beta}^{\gamma}\right)$. Thus, $d \Theta$ is contained in the ideal generated by $\left\{\Omega_{\alpha}^{\beta} \mid n<\alpha, \beta \leqq n+\nu\right\}$ is the exterior algebra of forms on $P \times \mathrm{SO}(n+\nu)$. On the other hand, $d \Theta$ can be dumped to $B$ and Lemma 2.2 applies to $\Theta$; in particular, $\Theta$ does not "contain" $\Omega_{\alpha}^{\beta}$. Thus $d \Theta=0$. Hence $d \Theta_{B}=0$. We have proved :

Proposition 4.1. Let $\Theta_{B}$ be the $\nu$-form on $B$ as defined above when $v$ is even. Then for an isometric immersion $f: M \rightarrow \boldsymbol{R}^{n+v}$, the form $f^{B^{*}} \Theta$ is a closed $\nu$-form on $M$ and the corresponding cohomology class is a regular homotopy invariant.

COROLLARY 4.2. If $\nu$ is even and $f$ is regularly homotopic to an isometric immersion of $M$ into $\boldsymbol{R}^{n+v-1}$, then $f^{B^{*} \Theta}$ is cohomologous to zero.

Roughly speaking, this is because we then have $\Omega_{n+v}^{i}=0$.

The rest of this section is devoted to several comments on $\Theta_{B}$ without proofs.

REMARK 4.1. $f^{B^{*} \Theta}$ is essentially the Euler class of the normal bundle of $f(M)$ in $\boldsymbol{R}^{n+v}$ (or rather of $f$ ). (See the next paper of this series for the Euler class of a differentiable vector bundle.) The corollary is obvious. The cohomology class $f^{B^{*}} \Theta_{B}$ is zero when $f$ is an imbedding (see Husemoller [5, p. 261] or the next paper). It is possible to express the Pontrjagin classes (see Chern [1]) of the normal bundle with the forms $\left(\Omega_{\alpha}^{i}\right)$. However, they are trivial since they are determined by the Pontrjagin classes of $M$ (due to the duality theorem) and independent of $f$ a priori.

REMARK 4.2. The invariant $f^{B^{*}} \Theta_{B}$ has an important meaning from the standpoint of obstruction theory (see Steenrod [9] for details). The fiber of $B$ is the Stiefel manifold $V_{n+v, n}$, and $(\nu-1)$-connected. And the $\nu$ th homotopy group $\pi_{\nu}\left(V_{n+v, n}\right)$ is isomorphic with $\boldsymbol{Z}$ or $\boldsymbol{Z}_{2}$ according as $\nu$ is even or odd. Now the odd case is out of our framework. When $v$ is even, $\pi_{v}\left(V_{n+v, n}\right)$ is naturally contained in $H_{i}\left(V_{n+v, n}\right)$ 
$\cong H^{i}\left(V_{n+v, n}\right)$ and spans this over $R$ by the Hurewicz theorem. It follows that the primary difference of two sections of $B$ appear in the cohomology group $H^{v}(M)$ over $H^{v}\left(V_{n+v, n}\right) \cong \boldsymbol{R}$, since $V_{n+v, n}$ is a homogeneous space, which is nice in a certain sense. Given two sections $s_{1}$ and $s_{2}$ of $B$, the cohomology class $s_{1}^{*} \Theta_{B}-s_{2}^{*} \Theta_{B} \in H^{2}(M)$ will essentially give their primary difference.

REMARK 4.3. When $n=\nu$ and is even, $f^{B^{*}} \Theta_{B}$ is exactly the only regular homotopy invariant (Hirsch [5]).

Remark 4.4. Apart from topology, some interest of Proposition 4.1 would lie in the fact that the homotopy invariant $f^{B^{*}} \Theta_{B}$ is completely described by the "second fundamental form" $\left(\Omega_{\alpha}^{i}\right)$ of $f$ (hence by the second order jet of $f$ ).

5. Some isometric homotopy invariants. In this section we will construct some isometric homotopy invariants. They are, as defined in the Introduction, cohomology classes on $M$ for each isometric immersion which remain constant under any smooth homotopy $F: M \times I \rightarrow \boldsymbol{R}^{n+v}$ such that each $f_{t}: M \rightarrow \boldsymbol{R}^{n+v}$ is an isometric immersion for $t \in[0,1]$, where $f_{t}(x)=F(x, t)$.

Even if it is not closed, a differential form $\Omega$ on $B$ will give rise to an isometric homotopy invariant $f_{\mathbf{E}}^{B^{*}} \Omega$ for each isometric immersion $f: M \rightarrow \boldsymbol{R}^{n+v}$ provided that $f^{B^{*}} \Omega$ is closed on $M \times I$ for any isometric homotopy $f: M \times I \rightarrow R^{n+\nu}$, where $F^{B}(x, t)=f_{t}^{B}(x)$. In fact, we then have, for each cycle $c$ in $M, \int_{c} f_{1}^{B^{*}} \Omega-\int_{c} f_{2}^{B^{*}} \Omega$ $=\int_{c \times\{1\}} F^{B^{*}} \Omega-\int_{c \times\{0\}} F^{B^{*}} \Omega= \pm \int_{\partial(c \times I)} F^{B^{*}} \Omega= \pm \int_{c \times I} d F^{B^{*}} \Omega=0$ by Stokes theorem, where $\partial$ denotes the boundary operator. Thus, what we are looking for is an $\Omega$ of degree $p$ on $B$ such that

$$
d \Omega\left(F_{*}^{B}(Y), F_{*}^{B}\left(X_{1}\right), \ldots, F_{*}^{B}\left(X_{p}\right)\right)=0
$$

for $Y, X_{1}, \ldots, X_{p} \in T_{(x, t)}(M \times I),(x, t) \in M \times I$. Here we may assume that $X_{1}, \ldots, X_{p}$ are tangent to $M \times\{t\}$. Then $F_{*}^{B}\left(X_{k}\right)=f_{t^{*}}^{B}\left(X_{k}^{\prime}\right), 1 \leqq k \leqq p$, where $X_{k}^{\prime}$ is the image of the natural isomorphism $T_{(x, t)}(M \times\{t\}) \cong T_{x}(M)$. By Proposition 3.2, these $f_{t^{*}}^{B}\left(X_{k}\right)$ belong to a holonomic horizontal space $f_{t^{*}}^{B}\left(T_{x}(M)\right)$. Therefore (5.1) follows from

$$
\iota(Y) \cdot d \Omega=0
$$

on any holonomic horizontal plane $H \subset T_{b}(B)$ and any vector $Y \in T_{b}(B)$ (see $\S 3$ ). In other words, we want to construct forms $\Omega$ on $B$ which is closed on each integral element $H$ of $\mathscr{H}$ and also closed in each subspace of the tangent space $T_{b}(B)$ which contains $H$ as a hyperplane.

Hereafter we consider the case where $\nu=1$ and $p=n$. As in the previous section, we first construct forms on $P \times \mathrm{SO}(n+1)=P \times V_{n+1, n}$ and then dump them down to $B$. Let $\Omega=\Omega(r)$ be the $n$-form, parametrized by $r \in R ; \Omega=\left(\omega^{1}+r \Omega_{n+1}^{1}\right)$ $\wedge\left(\omega^{2}+r \Omega_{n+1}^{2}\right) \wedge \cdots \wedge\left(\omega^{n}+r \Omega_{n+1}^{n}\right)$, where $\omega^{i}$ and $\Omega_{n+1}^{i}$ are the forms defined in $\S 2 . \Omega$ is invariant under the right action of $\mathrm{SO}(n) \times \mathrm{SO}(v)=\mathrm{SO}(n) \times \mathrm{SO}(1)=\mathrm{SO}(n)$, the proof being the same as for the volume element $\omega=\omega^{1} \wedge \omega^{2} \wedge \cdots \wedge \omega^{n}$ under SO $(n) . \Omega$ is transversal to the fibers of the bundle $P \times \mathrm{SO}(n+1) \rightarrow B$ since $\Omega$ 
does not contain $\omega_{j}^{i}-\Omega_{j}^{i}$, in view of Lemma 2.2. Therefore we can dump $\Omega$ to $B$ to obtain $\Omega_{B}=\Omega_{B}(r)$.

THEOREM 5.1. The form $\Omega_{B}$ on $B$ defined above gives rise to an isometric homotopy invariant $f^{B^{*}} \Omega_{B} \in H^{n}(M ; R)$ for any isometric immersion $f: M \rightarrow R^{n+1}$ and for any value of the parameter.

Proof. We have only to verify (5.2) for $\Omega_{B}$. We use the notations and the results in $§ 3$. (5.2) is equivalent to

$$
\iota\left(Y_{0}\right) d \Omega=0 \text { on } H_{0}
$$

for any tangent vector $Y_{0}$ in $T_{(p, v)}\left(P \times V_{n+1, n}\right)$ which contains the arbitrary $H_{0}$ in Lemma 3.4. Simply by taking the exterior derivative we obtain

$$
d \Omega=\sum_{i=1}^{n}(-1)^{i-1}\left(\omega^{1}+r \Omega_{n+1}^{1}\right) \wedge \cdots \wedge d\left(\omega^{i}+r \Omega_{n+1}^{i}\right) \wedge \cdots \wedge\left(\omega^{n}+r \Omega_{n+1}^{n}\right)
$$

and $d\left(\omega^{i}+r \Omega_{n+1}^{i}\right)=\sum_{j=1}^{n}\left(\omega^{j} \wedge \omega_{j}^{i}+r \Omega_{n+1}^{j} \wedge \Omega_{j}^{i}\right)$ by (2.4), (2.5) and (2.3). Since $\omega_{j}^{i}=\Omega_{j}^{i}=0$ on $H_{0}$ by Lemma 3.4 , we see $d\left(\omega^{i}+r \Omega_{n=1}^{i}\right)=0$ on $H_{0}$. Thus, if we put $y_{j}^{i}=\omega_{j}^{i}\left(Y_{0}\right)$ and $Y_{j}^{i}=\Omega_{j}^{i}\left(Y_{0}\right)$, we obtain

$$
\begin{aligned}
\iota\left(Y_{0}\right) d \Omega=-\sum_{i=1}^{n}\left(\omega^{1}+\right. & \left.r \Omega_{n+1}\right) \wedge \cdots \\
& \wedge\left(\sum_{j=1}^{n}\left(y_{j}^{i} \omega^{j}+r Y_{j}^{i} \Omega_{n+1}^{j}\right)\right) \wedge \cdots \wedge\left(\omega^{n}+r \Omega_{n+1}^{n}\right) .
\end{aligned}
$$

We note in (5.4) that the summation $\sum_{j}$ for $j$ ranges over the indices different from $i$ since both $\left(y_{j}^{i}\right)$ and $\left(Y_{j}^{i}\right)$ are skew-symmetric matrices. Now we use the following fact: if we choose a $(p, v) \in P \times V_{n+1, n}$ with $\pi(p, v)=b$ for a given $b \in B$ then there exist real numbers $c^{i}$ such that we have

$$
\left.\Omega_{n+1}^{i}=c^{i} \omega^{i}, \quad 1 \leqq i \leqq n, \quad \text { (not summed up for } i\right)
$$

on $H_{0}$ at $(p, v)$. We postpone the proof of (5.5) until the end of this section in order not to interrupt the stream of the proof of the theorem. (5.5) implies that we have (on $H_{0}$ )

$$
\omega^{i}+r \Omega_{n+1}^{i}=\left(1+r c^{i}\right) \omega^{i},
$$

and

$$
\sum_{j}\left(y_{j}^{i} \omega^{j}+r Y_{j}^{i} \Omega_{n+1}^{j}\right)=\sum_{j \neq 1}\left(y_{j}^{i} c^{j}+r Y_{j}^{i} c^{j}\right) \omega^{j} .
$$

Substituting (5.6) and (5.7) into (5.4), we conclude that each term of the righthand side of (5.4) vanishes since clearly $\omega^{1} \wedge \cdots \wedge \omega^{i-1} \wedge \omega^{j} \wedge \omega^{i+1} \wedge \cdots \wedge \omega^{n}=0$ for $j \neq i$. Theorem 5.1 is proved.

REMARK 5.1. Since $\Omega=\Omega(r)$ is a polynomial in $r$, so is $\Omega_{B}=\Omega_{B}(r)$. Let $\Omega_{(k)}$ be the coefficient of $r^{k}$ in $\Omega_{B}(r)$. Then Theorem 5.1 says that each $f^{B^{*}} \Omega_{(k)}$ is an isometric homotopy invariant for an isometric immersion $f: M \rightarrow \boldsymbol{R}^{n+1}$. On the other hand, 
we have $f^{B^{*}} \Omega(k)=s_{k} \omega$ if $s_{k}$ denotes the $k$ th elementary symmetric function of the principal curvatures $c^{1}, c^{2}, \ldots, c^{n}$ for $f$ and $\omega$ denote the volume element of $M$. Thus, Theorem 5.1 is equivalent to saying that the integral over $M$ of the elementary symmetric functions of the principal curvatures are isometric homotopy invariants. It is easy to see, however, that $f^{B^{*}} \Omega_{(k)}$ for even $k$ is determined by the Riemannian metric (and its curvature) of $M$ so that it is independent of $f$; in fact, $\Omega_{n+1}^{i} \wedge \Omega_{n+1}^{j}$ $=d \Omega_{i}^{j}-\sum_{k=1}^{n} \Omega_{k}^{i} \wedge \Omega_{k}^{j}=d \omega_{i}^{j}-\sum \omega_{k}^{i} \wedge \omega_{k}^{j}=K_{i}^{j}\left(\right.$ on $\left.U_{(p, v)}\right)$ by the formulas in preceding sections.

Remark 5.2 (SEE Chern [3] oR Weyl [11]). The integral $\int_{M} f^{B^{*}} \Omega_{B}$ has a very clear geometric meaning. Suppose for simplicity that $f$ is an isometric imbedding. Then that integral is the area of the hypersurface $M_{r}$, defined for small $|r|$, consisting of the points in $R^{n+1}$ at the distance $|r|$ from $f(M)$ in a certain side of $f(M)$, or equivalently, $\left|\int_{0}^{r} d r \int_{M} f^{B^{*}} \Omega_{B}\right|$ is the volume of the "annular" domain between $f(M)$ and $M_{r}$. This interpretation allows us to generalize the theorem to the case of arbitrary codimensions $\nu$. But if $\nu>1$, resulting invariant is determined by the curvature of $M$ alone, as is known since $\mathrm{H}$. Weyl.

REMARK 5.3. $f^{B^{*}} \Omega_{(n)}$, or rather its integral over $M$, is essentially the degree of the Gauss map. Thus, if $f$ is an imbedding and $n=\operatorname{dim} M$ is even, $f^{B^{*}} \Omega_{(n)}$ is essentially the Euler class of $M$ by Hopf's theorem. Thus the Gauss-Bonnet theorem is obtained by expressing $f^{B^{*}} \Omega_{B}$ with the curvature. However, this is the only (topological) invariant among $f^{B^{*}} \Omega_{(k)}, 0 \leqq k \leqq n$, that is independent of the Riemannian metric of $M$. To see this, take $M$ to be the hypersphere of radius $a$ in $R^{n+1}$ and let $f$ be the inclusion map. Then we have $\omega^{i}=a \Omega_{n+1}^{i}$ and hence $f^{B^{*}} \Omega_{B}=(1+(r / a))^{n} \omega$, where $\omega$ is the volume element.

REMARK 5.4. As regards the existence (or nonexistence) of isometric homotopies, we should note the following: If the second fundamental form is of rank $\geqq 3$, at any point of $M$, then the isometric immersion of the hypersurface $M$ is rigid (T. Y. Thomas [10]). There always exists such a point for a compact $M$ (Chern [2]). This kind of isometric homotopies is of extremely restricted type if one admits the result of [4].

REMARK 5.5. The theorem is also true for a compact hypersurface with boundary if the homotopy fixes the boundary pointwise.

Proof of (5.5). First we derive a formula similar to (3.3) in a similar way:

$$
\begin{aligned}
\sum_{\lambda} V_{\alpha}^{\lambda} d \Omega^{\lambda} & =d\left(\sum_{\lambda} V_{\alpha}^{\lambda} \Omega^{\lambda}\right)-\sum d V^{\lambda} \wedge \Omega^{\lambda} \\
& =d\left(\sum_{\lambda} V_{\alpha}^{\lambda} \sum_{i} V_{i}^{\lambda} \omega^{i}\right)-\sum_{\lambda} d V_{\alpha}^{\lambda} \wedge \sum_{i}\left(V_{i}^{\lambda} \omega^{i}\right) \\
& =\sum_{i} d\left(\left(\sum_{\lambda} V_{\alpha}^{\lambda} V_{i}^{\lambda}\right) \omega^{i}\right)-\sum_{i}\left(\sum_{\lambda} V_{i}^{\lambda} d V_{\alpha}^{\lambda}\right) \wedge \omega^{i}=\sum_{i} d\left(\delta_{i}^{\alpha} \omega^{i}\right)-\sum_{i} \Omega_{i}^{\alpha} \wedge \omega^{i} \\
& =0+\sum \Omega_{\alpha}^{i} \wedge \omega^{i}
\end{aligned}
$$


on $P \times \mathrm{SO}(n+\nu)$ for $\alpha>n$. Since $d \Omega^{\lambda}=0$ on $H_{0}, \sum \Omega_{\alpha}^{i} \wedge \omega^{i}=0$ follows from the above. As in the proof of Lemma 3.5, we have $\Omega_{\alpha}^{i}=\sum_{j} A_{i}^{\alpha} j \omega^{j}$ with $A_{j i}^{\alpha}=A_{i j}^{\alpha}$ on $H_{0}$. Now let $\nu=1$ and hence $\alpha=n+1$. We have a symmetric form $\left(A_{i j}^{n+1}\right)$. Let $(p, v)$ be the origin of $H_{0}$. Put $\pi(p, v)=b$. It is easy to see that $\left(A_{i j}^{n+1}\right)$ is sent to ${ }^{t} g\left(A_{i j}^{n+1}\right) g$ by the transformation $g$ sending $(p, v)$ to $(p g, v g)$. Thus, by a suitable choice of $g$, we can diagonalize ${ }^{t} g\left(A_{i j}^{n+1}\right) g$ so that we have $\left(A_{i j}^{n+1}\right)=\left(\delta_{i j} c_{j}\right)$, or $\Omega_{n+1}^{i}=c^{i} \omega^{i}$, for $H_{0} g \subset T_{c p g, v g}\left(P \times V_{n+1, n}\right)$.

\section{BIBLIOGRAPHY}

1. S. S. Chern, On curvature and characteristic classes of a Riemann manifold. Abh. Math. Sem. Univ. Hamburg 20 (1955), 117-126.

2. —, Topics in differential geometry, Inst. for Advanced Study, Princeton, N. J. (mimeographed).

3. — Lecture on integral geometry, Summer Science Seminar (Academia Sinica, Taiwan Univ. and Tsing Hua Univ.), 1965.

4. S. Dolbeault-Lemoine, Sur la déformabilité des variétés plongées dans un espace de Riemann, Ann. Sci. École Norm. Sup. 73 (1956), 357-438.

5. M. W. Hirsch, Immersions of manifolds, Trans. Amer. Math. Soc. 93 (1959), 242-276.

6. D. Husemoller, Fiber bundles, McGraw-Hill, New York, 1966.

7. S. Kobayashi, Induced connections and imbedded Riemannian spaces, Nagoya Math. J. 10 (1956), 15-25.

8. W. F. Pohl, The self-linking number of a closed space curve, (to appear).

9. N. E. Steenrod, The topology of fibre bundles, Math. Note Series, Vol. 14, Princeton Univ. Press, Princeton, N. J., 1951.

10. T. Y. Thomas, Riemann spaces of class one and their characterization, Acta Math. 67 (1936), 169-211.

11. H. Weyl, On the volume of tubes, Amer. J. Math. 61 (1939), 461-472.

12. H. Whitney, Geometric integration, Princeton Univ. Press, Princeton, N. J., 1957.

University of Notre Dame,

Notre Dame, Indiana 